\title{
Unusual transformations of 1-vinyl-4,5,6,7-tetrahydro-5-methyl-4,6- ethanopyrrolo[3,2-c]pyridine to cyclohepteno[b]pyrroles under acetylation conditions
}

\author{
Alexey V. Varlamov, Tatiana N. Borisova, Leonid G. Voskressensky, Alla A. Brook, and \\ Alexey I. Chernyshev
}

Organic Chemistry Department of the Russian Peoples Friendship University, 3, Ordzhonikidze St., 117419 Moscow, Russia

E-mail:rpfu@orc.ru

\begin{abstract}
Heating 1-vinyl-4,5,6,7-tetrahydro-5-methyl-4,6-ethanopyrrolo[3,2-c]pyridine (1) in the presence of acetic or trifluoroacetic anhydride resulted in bicyclic saturated fragment cleavage, affording cyclohepteno[b]pyrroles 2a,b in low yields. In the case of trifluoroacetic anhydride, the major product of the reaction, -trifluoroacetylsubstituted tetrahydropyrrolo[3,2-c]pyridine 3 was isolated in $34 \%$ yield.
\end{abstract}

Keywords: Cyclohepteno[b]pyrroles, trifluoroacetic anhydride, acetylation

\section{Introduction}

Recently we have reported piperidine ring cleavage in tetrahydropyrrolo[3,2-c]pyridines under the action of acetic anhydride at $70{ }^{\circ} \mathrm{C}$ resulting with the formation of 2 - $(\alpha-$ methyl- $\beta$ acetamidoethyl)-3-vinylpyrroles in moderate yields, while the target 2-acetyl substituted pyrroles have not been isolated. ${ }^{1}$

We have studied the reactions of acetylation and trifluoroacetylation of $N$-vinylsubstituted tetrahydropyrrolo[3,2-c]pyridine 1 with bicyclic saturated fragment, synthesized by applying the Trofimov reaction to the oxime of tropinone.

The reaction of 1 with acetic anhydride at $70{ }^{\circ} \mathrm{C}$ leads to the bicyclic fragment cleavage at $\mathrm{C}_{4}-\mathrm{N}$ bond affording cyclohepteno[b]pyrrole $2 \mathrm{a}$ in $12 \%$ yield. The reaction was accompanied by significant tarring and there was no observable trace of $\alpha$-acetyl substituted tetrahydropyrrolo[3,2-c]pyridine which would have resulted from pyrrole fragment acetylation.

Treating tetrahydropyrrolopyridine 1 with trifluoroacetic anhydride under the same reaction conditions, as distinct from the reaction of 4,5,6,7-tetrahydro-4,5,7-trimethylpyrrolo[3,2c]pyridine derivatives, ${ }^{1}$ also led to the bicyclic saturated moiety opening giving cyclohepteno[b]pyrrole $2 \mathrm{~b}$ in $16 \%$ yield. The major product of this reaction, $\alpha$ trifluoroacetylsubstituted tetrahydropyrrolo[3,2-c]pyridine 3 was isolated in $34 \%$ yield. 
Cyclohepteno[b]pyrroles 2a,b exist as mixtures of two isomers due to the amide fragment hindrance. The alternative cyclohepteno[b]pyrroles 4 , that could have been formed as the result of the $\mathrm{C}_{6}-\mathrm{N}$ bond cleavage, were not detected.

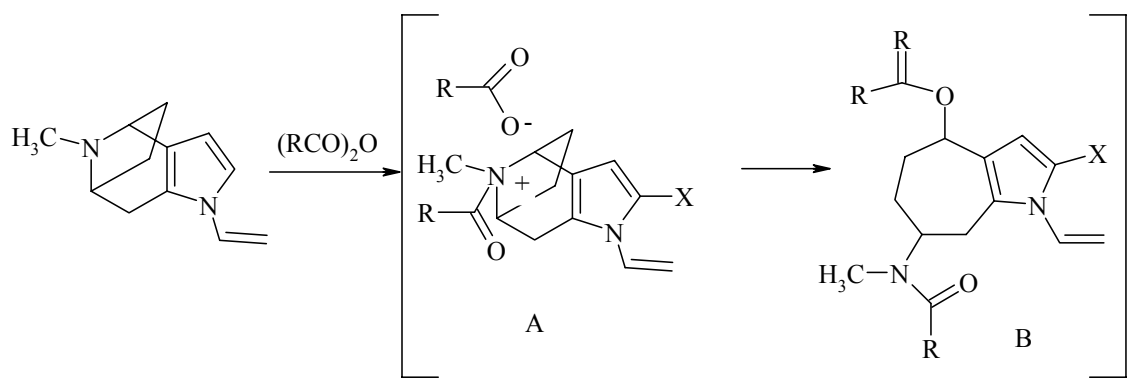

I

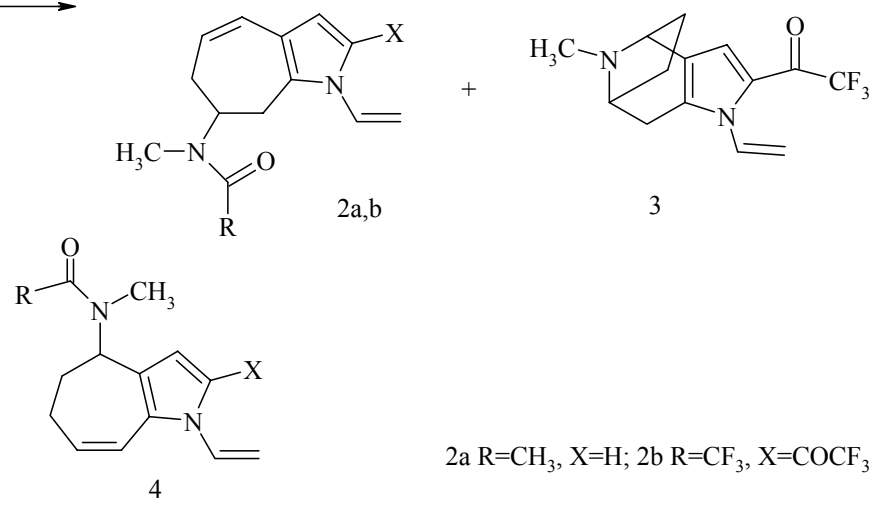

The reaction seems to proceed through the intermediacy of quaternary salt $\mathrm{A}$, which then predictably is cleaved by the acyloxy anion. This transformation can proceed either like Brown ${ }^{4}$ or Hofmann ${ }^{5}$ reaction, however the current experimental material does not permit to make a definite conclusion about the mechanism. Further work aimed at exploring the scope and limitations of this process is under way.

\section{Experimental Section}

General Procedures. Melting points are uncorrected. IR spectra of samples were obtained as $\mathrm{KBr}$ pellets. ${ }^{1} \mathrm{H}$ NMR chemical shifts are given as values with reference to $\mathrm{Me}_{4} \mathrm{Si}$ as internal standard. All column chromatography was carried out on Fluka aluminum oxide (activated, II grade, 150 mesh) at medium pressure (200 mbar). All solvents were distilled before use.

1-Vinyl-7-( $N$-methyl- $N$-acetylamino)cyclohept-4-ene[b]pyrrole (2a). To a stirred solution of tetrahydropyrrolo[3,2-c]pyridine $1(0.45 \mathrm{~g}, 2.4 \mathrm{mmol})$ in $40 \mathrm{~mL}$ of anhydrous toluene, freshly distilled acetic anhydride $(2.3 \mathrm{~mL}, 24 \mathrm{mmol})$ was added dropwise. The solution was stirred for 2 
$\mathrm{h}$ at $70{ }^{\circ} \mathrm{C}$, the excess of acetic anhydride and toluene were distilled off under reduced pressure. The residue was treated with saturated aqueous potassium carbonate $(50 \mathrm{~mL})$ and extracted with dichloromethane $(4 \times 50 \mathrm{~mL})$.

The organic extract was dried over magnesium sulfate. Evaporation of the solvent under reduced pressure left an oily residue, which was purified by column chromatography (heptane: ethyl acetate, 5:1), affording compound $2 \mathrm{a}$ in $12 \%$ yield as a mixture of isomers (52:48), pale-yellow solid: $\mathrm{mp} 129-130{ }^{\circ} \mathrm{C}$ (ethyl acetate-heptane); ${ }^{1} \mathrm{H}$ NMR $\left(200 \mathrm{MHz}, \mathrm{CDCl}_{3}\right.$ ) major isomer $\delta 2.1$ (s, 3H), $2.94(\mathrm{~s}, 3 \mathrm{H}), 2.2-3.2(\mathrm{~m}, 4 \mathrm{H}), 4.68(\mathrm{dd}, 1 \mathrm{H}, J=8.5$ and $0.9 \mathrm{~Hz}), 4.94(\mathrm{~m}, 1 \mathrm{H}), 5.08(\mathrm{dd}$, $1 \mathrm{H}, J=15.6$ and $0.9 \mathrm{~Hz}), 5.55(\mathrm{~m}, 1 \mathrm{H}), 6.05(\mathrm{~d}, 1 \mathrm{H}, J=3.1 \mathrm{~Hz}), 6.20(\mathrm{dd}, 1 \mathrm{H}, J=11.3$ and 3.1 $\mathrm{Hz}), 6.79(\mathrm{dd}, 1 \mathrm{H}, J=8.5 \mathrm{~Hz}, 15.6 \mathrm{~Hz}), 6.88(\mathrm{~d}, 1 \mathrm{H}, J=3.1 \mathrm{~Hz})$; minor isomer $\delta 2.12(\mathrm{~s}, 3 \mathrm{H})$, $2.89(\mathrm{~s}, 3 \mathrm{H}), 2.2-3.2(\mathrm{~m}, 4 \mathrm{H}), 4.76(\mathrm{dd}, 1 \mathrm{H}, J=8.5$ and $0.9 \mathrm{~Hz}), 4.24(\mathrm{~m}, 1 \mathrm{H}), 5.13(\mathrm{dd}, 1 \mathrm{H}, J=$ 15.6 and $0.9 \mathrm{~Hz}), 5.55(\mathrm{~m}, 1 \mathrm{H}), 6.08(\mathrm{~d}, 1 \mathrm{H}, J=3.1 \mathrm{~Hz}), 6.24(\mathrm{dd}, 1 \mathrm{H}, J=11.3$ and $3.1 \mathrm{~Hz}), 6.79$ (dd, $1 \mathrm{H}, J=8.5 \mathrm{~Hz}, 15.6 \mathrm{~Hz}), 6.92(\mathrm{~d}, 1 \mathrm{H}, J=3.1 \mathrm{~Hz})$; IR (KBr) $1650 \mathrm{~cm}^{-1}$; HRMS calcd for $\mathrm{C}_{14} \mathrm{H}_{18} \mathrm{~N}_{2} \mathrm{O} \mathrm{m} / \mathrm{z}$ 230.1419, found $\mathrm{m} / \mathrm{z}$ 230.1422; MS (m/z, rel. intensity) $230\left(\mathrm{M}^{+}, 13\right), 203(24)$, 180(11), 168(7), 157(100), 156(52), 144 (10), 130 (10). Anal. Calcld for $\mathrm{C}_{14} \mathrm{H}_{18} \mathrm{~N}_{2} \mathrm{O}: \mathrm{C}, 73.01$; H, 7.88; N, 12.16. Found: C, 73.32; H, 7.98; N, 12.41 .

\section{1-Vinyl-2-trifluoroacetyl-7-( $N$-methyl- $N$-trifluoroacetylamino)cyclohept-4-ene[b]pyrrole}

(2b) and 1-vinyl-2-trifluroacetyl-4,5,6,7-tetrahydro-5-methyl-4,6-ethanopyrrolo[3,2c]pyridine (3). Using the same procedure, tetrahydro-pyrrolo[3,2-c]pyridine 1 (0.46 g, $2.5 \mathrm{mmol})$ was treated with trifluoroacetic anhydride $(4.9 \mathrm{~g}, 25 \mathrm{mmol})$ at $70{ }^{\circ} \mathrm{C}$. Usual work-up gave oily residue, which was purified by column chromatography (heptane: ethyl acetate, 5:1), first providing compound $2 \mathrm{~b}$ as a mixture of two isomers $(60: 40)$ in $16 \%$ yield, green solid: $\mathrm{mp}$ $142-144{ }^{\circ} \mathrm{C}$ (heptane-ethyl acetate). ${ }^{1} \mathrm{H}$ NMR $\left(200 \mathrm{MHz}, \mathrm{CDCl}_{3}\right)$ major isomer $\delta 3.1(\mathrm{~s}, 3 \mathrm{H})$, $2.54-3.30(\mathrm{~m}, 4 \mathrm{H}), 4.70(\mathrm{tt}, 1 \mathrm{H}, J=10.7$ and $2.8 \mathrm{~Hz}), 5.16(\mathrm{~d}, 1 \mathrm{H}, J=15.9 \mathrm{~Hz}), 5.50(\mathrm{~d}, 1 \mathrm{H}, J=$ $8.5 \mathrm{~Hz}), 5.69(\mathrm{ddd}, 1 \mathrm{H}, J=11.9,7.0$ and $3.0 \mathrm{~Hz}), 6.27(\mathrm{dd}, 1 \mathrm{H}, J=11.9$ and $3.1 \mathrm{~Hz}), 7.08(\mathrm{q}, 1 \mathrm{H}$, $J=2.1 \mathrm{~Hz}), 7.15(\mathrm{dd}, 1 \mathrm{H}, J=8.5 \mathrm{~Hz}, 15.9 \mathrm{~Hz})$; minor isomer $\delta 3.1(\mathrm{~s}, 3 \mathrm{H}), 2.54-3.30(\mathrm{~m}, 4 \mathrm{H})$, $4.40(\mathrm{~m}, 1 \mathrm{H}), 5.14(\mathrm{~d}, 1 \mathrm{H}, J=15.9 \mathrm{~Hz}), 5.52(\mathrm{~d}, 1 \mathrm{H}, J=8.5 \mathrm{~Hz}), 5.69$ (ddd, $1 \mathrm{H}, J=11.9,7.0$ and $3.0 \mathrm{~Hz}), 6.29(\mathrm{dd}, 1 \mathrm{H}, J=11.9$ and $3.1 \mathrm{~Hz}), 7.06(\mathrm{q}, 1 \mathrm{H}, J=2.1 \mathrm{~Hz}), 7.14(\mathrm{dd}, 1 \mathrm{H}, J=8.5$ $\mathrm{Hz}, 15.9 \mathrm{~Hz}$ ); IR (KBr) $1680 \mathrm{~cm}^{-1}$; HRMS calcd for $\mathrm{C}_{16} \mathrm{H}_{14} \mathrm{~F}_{6} \mathrm{~N}_{2} \mathrm{O}_{2} \mathrm{~m} / \mathrm{z} 380.0959$, found $\mathrm{m} / \mathrm{z}$ 380.0951; MS ( $\mathrm{m} / \mathrm{z}$, rel. intensity) $380\left(\mathrm{M}^{+}, 8\right), 253(100), 252(25), 184(55), 156(50), 128(11)$, 127(10), 69(20); Anal. Calcld for $\mathrm{C}_{16} \mathrm{H}_{14} \mathrm{~F}_{6} \mathrm{~N}_{2} \mathrm{O}_{2}$ : C, 50.53; H, 3.71; N, 7.37. Found: C, 50.32; H, 4.02; N, 6.96 .

Continued elution of the chromatography column provided 3 as a yellow oil in $34 \%$ yield. ${ }^{1} \mathrm{H}$ NMR (200 MHz, CDCl $) \delta 1.48(\mathrm{~m}, 1 \mathrm{H}), 1.81(\mathrm{~m}, 1 \mathrm{H}), 2.1-2.3(\mathrm{~m}, 2 \mathrm{H}), 2.35(\mathrm{~s}, 3 \mathrm{H}), 2.40(\mathrm{~d}$, $1 \mathrm{H}, J=17.7 \mathrm{~Hz}), 3.15(\mathrm{dd}, 1 \mathrm{H}, J=17.7$ and $4.6 \mathrm{~Hz}), 3.57(\mathrm{~m}, 1 \mathrm{H}), 3.91(\mathrm{~d}, 1 \mathrm{H}, J=5.5 \mathrm{~Hz}), 5.18$ (dd, $J=15.9$ and $0.9 \mathrm{~Hz}), 5.20(\mathrm{dd}, 1 \mathrm{H}, J=8.9$ and $0.9 \mathrm{~Hz}), 7.02(\mathrm{q}, 1 \mathrm{H}, J=2.1 \mathrm{~Hz}), 7.41(\mathrm{dd}, J$ $=15.9$ and $8.9 \mathrm{~Hz})$; IR (KBr) $1690 \mathrm{~cm}^{-1}$; MS ( $\mathrm{m} / \mathrm{z}$, rel. intensity) $284\left(\mathrm{M}^{+}, 44\right), 269(6), 255(100)$, 243(5), 229(5), 157(6); Anal. Calcld for $\mathrm{C}_{14} \mathrm{H}_{15} \mathrm{~F}_{3} \mathrm{~N}_{2} \mathrm{O}: \mathrm{C}, 59.15$; H, 5.32; N, 9.85. Found: $\mathrm{C}$, $59.12 ; \mathrm{H}, 4.98 ; \mathrm{N}, 9.46$. 


\section{References}

1. Voskressensky, L. G.; Varlamov, A. V.; Borisova, T. N.; Sorokina, E. A. Book of Abstracts of the $17^{\text {th }}$ International Congress of Heterocyclic Chemistry Vienna 1999, Poster 300

2. Trofimov, B. A.; Mikhaleva, A. I. Heterocycles 1994, 37, 1193.

3. Varlamov, A. V.; Borisova T. N.; Voskressensky, L. G.; Levov, A. N.; Chernyshev, A. I. Khim. Geterotsikl. Soedin. 1999, 5, 610.

4. Vaughan, W. R.; Carlson, R. D. J. Am. Chem. Soc. 1962, 84, 769.

5. Hinskey, R. G. J. Org. Chem. 1964, 29, 3678. 